\title{
N-type self-assembled monolayer field-effect transistors
}

\author{
Andreas Ringk ${ }^{\mathrm{a}, \mathrm{b}}$, Xiaoran $\mathrm{Li}^{\mathrm{c}, \mathrm{d}}$, Fatemeh Gholamrezaie ${ }^{\mathrm{b}, \mathrm{e}, \mathrm{f}}$, Edsger C. P. Smits ${ }^{\mathrm{c}}$, Alfred Neuhold ${ }^{\mathrm{g}}$, \\ Armin Moser ${ }^{\mathrm{g}}$, Gerwin H. Gelinck ${ }^{\mathrm{c}}$, Roland Resel ${ }^{\mathrm{g}}$, Dago M. de Leeuw ${ }^{\mathrm{e}, \mathrm{f}}$, Peter Strohriegl ${ }^{{ }^{\mathrm{a}}}$ \\ ${ }^{a}$ Macromolecular Chemistry I, University of Bayreuth, Universitätsstrasse 30, \\ 95440 Bayreuth, Germany; \\ ${ }^{\mathrm{b}}$ Dutch Polymer Institute (DPI), P.O. Box 902, 5600 AX Eindhoven, The Netherlands; \\ ${ }^{\mathrm{c}}$ Holst Centre/TNO, High Tech Campus 31, 5656 AE Eindhoven, The Netherlands; \\ ${ }^{\mathrm{d}}$ Department of Chemical Engineering and Chemistry, Eindhoven University of Technology, P.O. \\ Box 513, 5600 MB Eindhoven, The Netherlands; \\ ${ }^{\mathrm{e}}$ Philips Research Laboratories, High Tech Campus 4, 5656 AE Eindhoven, The Netherlands; \\ ${ }^{\mathrm{f}}$ Molecular Electronics, Zernike Institute for Advanced Materials, University of Groningen, \\ Nijenborgh 4, 9747 AG Groningen, The Netherlands; \\ ${ }^{\mathrm{g}}$ Institute of Solid State Physics, Graz University of Technology, Petersgasse 16, A-8010 Graz
}

\begin{abstract}
Within this work we present the synthesis and applications of a novel material designed for n-type self-assembled monolayer field-effect transistors (SAMFETs). Our novel perylene bisimide based molecule was obtained in six steps and is functionalized with a phosphonic acid linker which enables a covalent fixation on aluminum oxide dielectrics. The organic field-effect transistors (OFETs) were fabricated by submerging predefined transistor substrates in a dilute solution of the molecule under ambient conditions. Investigations showed a thickness of about $3 \mathrm{~nm}$ for the organic layer which is coincides to the molecular length. The transistors showed bulk-like electron mobilities up to $10^{-3} \mathrm{~cm}^{2} / \mathrm{Vs}$. Due to the absence of bulk current high on/off-ratios were achieved. An increase of the electron mobility with the channel length and XPS investigations point to a complete coverage of the dielectric with a dense monolayer. In addition, a p-type SAMFET based on a thiophene derivative and our new n-type SAMFET were combined to the first CMOS bias inverter based solely on SAMFETs.
\end{abstract}

Keywords: Self-assembled monolayer, n-type field-effect transistor, perylene bisimide, organic circuits, complementary inverter

\section{INTRODUCTION}

Self-assembly is the spontaneous and autonomous organization of molecules into patterns ${ }^{1}$. Self-assembling molecules usually contain an organic tail with one reactive group which is able to bind covalently to a surface. Dense and complete coverage can be achieved over large areas on a short time scale. The organic tails passivate the surface so that in many cases only one single monolayer is formed. Depending on the surface different anchor groups are used. The best known monolayers are thiols on gold. Organic acids or chlorosilanes are often used to modify inorganic oxides whereas silicon

*Peter.Strohriegl@uni-bayreuth.de; phone+49 92155 3207; http://www.macro1.de

Organic Field-Effect Transistors XI, edited by Zhenan Bao, lain McCulloch, Proc. of SPIE Vol. 8478

$847813 \cdot$ ○ 2012 SPIE $\cdot$ CCC code: 0277-786/12/\$18 · doi: 10.1117/12.929535 
can be modified with materials having terminal double bonds by hydrosilylation ${ }^{2}$. In such a way a monolayer is able to change the reactivity or polarity in order to passivate or protect the material surface.

Because the formation of self-assembled monolayers (SAMs) is a simple and quick method to achieve new material properties this technique arrived early in the broad field of organic semiconducting devices. In organic solar cells monolayers of dye molecules on titanium dioxide are used for the absorption of light and an efficient charge transfer ${ }^{3}$. In organic light emitting diodes monolayers are used to modify indium tin oxide ${ }^{4}$. Also in organic field effect transistors (OFETs) SAMs are used to improve the performance. Common transistor substrates are based on silicon with a layer of silicon dioxide on top which is used as the dielectric. On this surface polar hydroxyl groups are present which have a negative influence on the charge transport especially for electrons ${ }^{5}$. In order to get rid of the polar groups, SAMs, for example chlorosilanes with long alkyl chains, are often used which react with the hydroxyl groups to create a non polar surface $^{[5]}$. The surface energy is also changing so that the monolayer can change the morphology of the semiconductor too ${ }^{6}$. It also has been shown that monolayers can be used as a thin dielectric resulting in transistors with very low operating voltages ${ }^{7}$. Because charge injection from the source electrode into the organic semiconductor plays a crucial role the electrodes, typically made of gold, can be covered with thiols in order to match the energy levels of the electrode and the organic semiconductor ${ }^{8}$.

It is known that charge transport in p- and n-type field-effect transistors occurs in an ultrathin accumulation layer close to the dielectric ${ }^{9,10}$. This finding spurred interest in having a transistor with only one layer of a semiconducting molecule fixed to the dielectric surface by simple self-assembly (see Figure 1). A so called self-assembled monolayer field-effect transistor (SAMFET) was first demonstrated by Tulevski et al. ${ }^{11}$. They attached a functionalized tetracene onto an aluminum oxide dielectric to form sub micron transistors. Meanwhile different other groups realized SAMFETs. Probably the best working SAMFETs are made from a thiophene derivative self-assembled onto a silicon dioxide surface $^{12}$. A dense, highly crystalline and well packed monolayer was formed to enable transistors with channel length up to $40 \mu \mathrm{m}$ and hole mobilities of $10^{-2} \mathrm{~cm}^{2} /$ Vs. Additionally the first integrated circuits such as a 15 bit code generator were demonstrated using this bottom-up approach. Because the active channel in SAMFETs is only a single layer no bulk current occurs, which results in high on/off ratios up to $10^{6}$.

State of the art integrated circuits are mostly based on the well-established complementary metal oxide semiconductor (CMOS) technique, where pairs of $\mathrm{p}$ - and n-type transistors are combined in one device. The main advantages of CMOScircuits are high noise immunity and low power consumption. Compared to p-type materials electron transporting transistors are more difficult to realize because of the high reactivity towards oxygen and water ${ }^{13}$. For CMOS circuits based on SAMFETs suitable n-type materials are needed. So far only Novak et al. showed n-type SAMFETs using a fullerene derivative ${ }^{14}$ with electron mobilities of $10^{-4} \mathrm{~cm}^{2} / \mathrm{Vs}$. However, no SAMFET based complimentary circuit has been demonstrated so far.

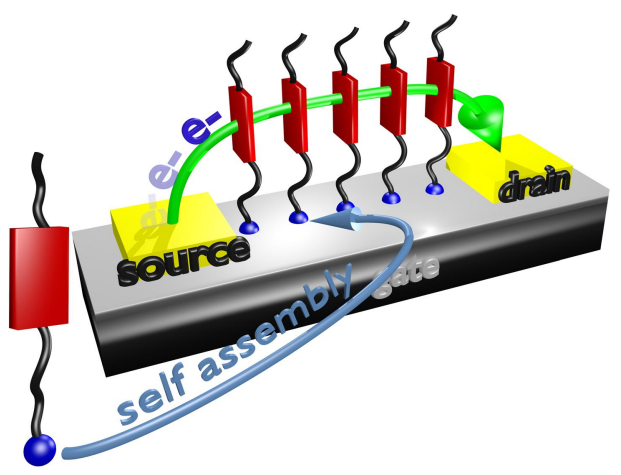

Figure 1: Schematic illustration of a self-assembled monolayer field effect transistor (SAMFET).

Here we present a promising n-type material for the use in self-assembled monolayer field-effect transistors. The novel material we used is based on a perylene bisimide (PBI) which is well known for thin film transistor applications ${ }^{15}$. For our purpose we synthesized a heterosubstituted perylene bisimide where a phosphonic acid is attached at one side of the molecule in order to enables a covalent fixation to an aluminum oxide dielectric. 


\section{SYNTHESIS}

Perylene bisimides (PBIs) are well known for their good performance in field effect transistors ${ }^{[15]}$. Typically homo substituted perylene bisimides are used where the perylene molecule has the same linear tail at both sides. The disadvantage of homo substituted perylene bisimides with linear tails are their low solubility in almost all solvents. To fabricate organic transistors with those perylene bisimides the physical vapor deposition technique is commonly used. High evaporation temperatures in a vacuum chamber are needed to sublime the material. Also the transistor substrates must be heated up to about $130^{\circ} \mathrm{C}$ to get high crystallinity for a good charge transport. In order to circumvent those high temperatures we synthesized a perylene bisimide with a branched alkyl tail on one side which increases the solubility and allows a solution based SAMFET preparation. To enable the fixation to an aluminum oxide dielectric a phosphonic acid group is introduced to the perylene bisimide. Whereas homosubstituted PBIs can be synthesized in high yield in only one step from perylene-3,4,9,10-tetracarboxylic dianhydride (PTCDA), heterosubstituted PBIs are more difficult to realize. In principle two synthetic strategies are known ${ }^{16}$ to synthesize heterosubstituted PBIs. One of these routes was used for our synthesis and will be described in detail in the following chapter ${ }^{17}$.

PTCDA 1 is used as the starting material which is refluxed in a potassium hydroxide solution. During this reaction both anhydride groups are cleaved. Titration with acetic acid leads to the monopottassium salt 2.

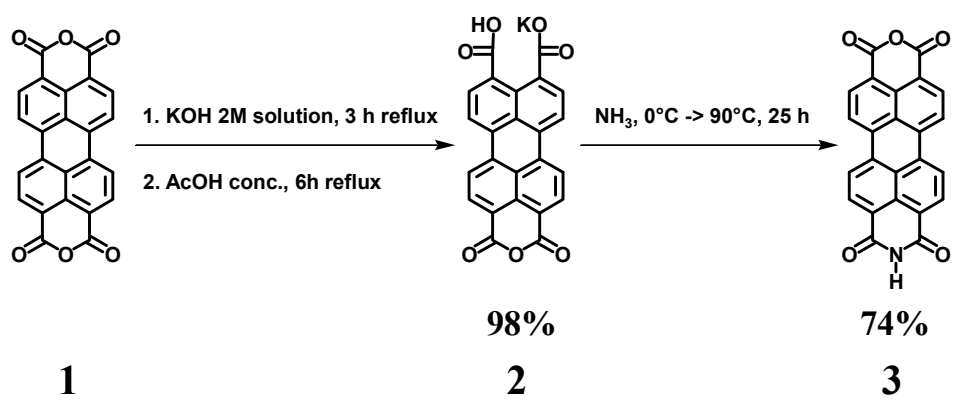

Figure 2: Synthetic strategy towards perylene monoimide anhydride.

The potassium salt $\mathbf{2}$ acts as a protective group so that in the following step ammonia reacts with the anhydride to form an imide group (Figure 2). After acidic workup the potassium group is transferred back into the anhydride to yield the asymmetric monoimide anhydride 3 .

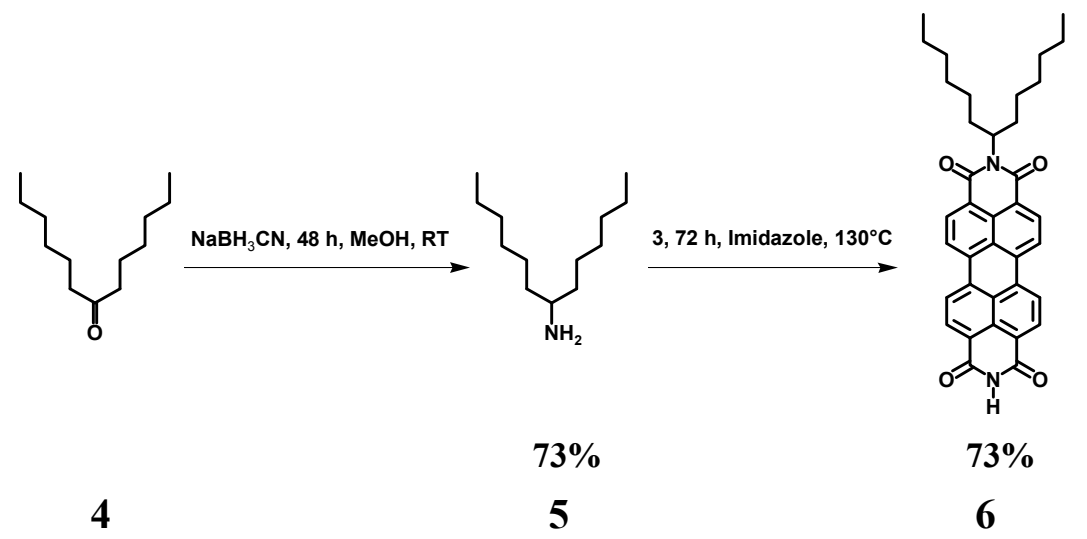

Figure 3: Synthetic strategy towards a heterosubstituted perylene bisimide.

Dihexylketone 4 reacts with sodium cyanoborohydride during a reductive amination reaction to the branched amine 5 $(\text { Figure } 3)^{18}$. In the following step the amine $\mathbf{5}$ reacts with the previously synthesized perylene monoimide anhydride $\mathbf{3}$ to the heterosubstituted perylene bisimide $\mathbf{6}$. Due to the electron withdrawing character of the perylene core the proton of the bisimide 6 directly attached to the nitrogen can be removed with sodium hydride (Figure 4). The resulting anion reacts in an $\mathrm{S}_{\mathrm{N}} 2$-reaction with an excess of 1,11-dibromoundecane to form bisimide 7 . 


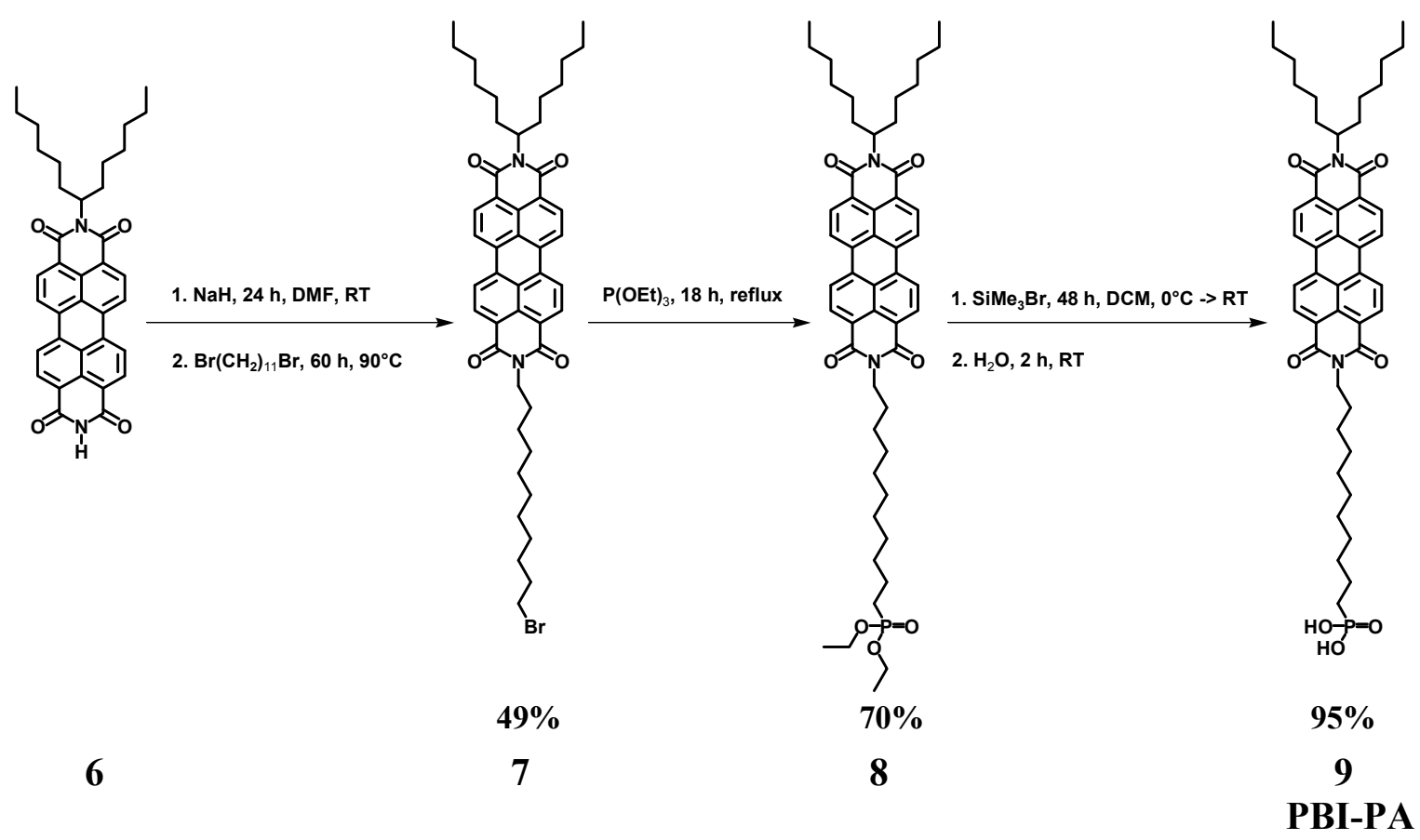

Figure 4: Synthetic strategy towards the target molecule PBI-PA 9.

The Michaelis-Arbuzow reaction was used to transform bromide 7 into the phosphonic ester 8. The phosphonic ester is cleaved using bromotrimethylsilane. After adding some water to the reaction mixture the phosphonic acid is generated and the target molecule PBI-PA 9 precipitates. Nuclear magnetic resonance (NMR) showed a complete conversion of the phosphonic ester 8 into the phosphonic acid PBI-PA 9. The overall yield of the reaction sequence from 1 to 9 is $17 \%$.

UV-vis absorption and fluorescence measurements of PBI-PA in THF solution are depicted in figure 5. The absorption spectrum shows a maximum at $519 \mathrm{~nm}$. Two additional blue-shifted vibrational satellites appear at 484 and $459 \mathrm{~nm}$. The absorption edge is at $539 \mathrm{~nm}$ from which a band gap of $2.30 \mathrm{eV}$ is calculated. After excitation at $484 \mathrm{~nm}$ the fluorescence spectrum shows an emission maximum at $529 \mathrm{~nm}$. A second red-shifted peak appears at $568 \mathrm{~nm}$ with a shoulder at $611 \mathrm{~nm}$.

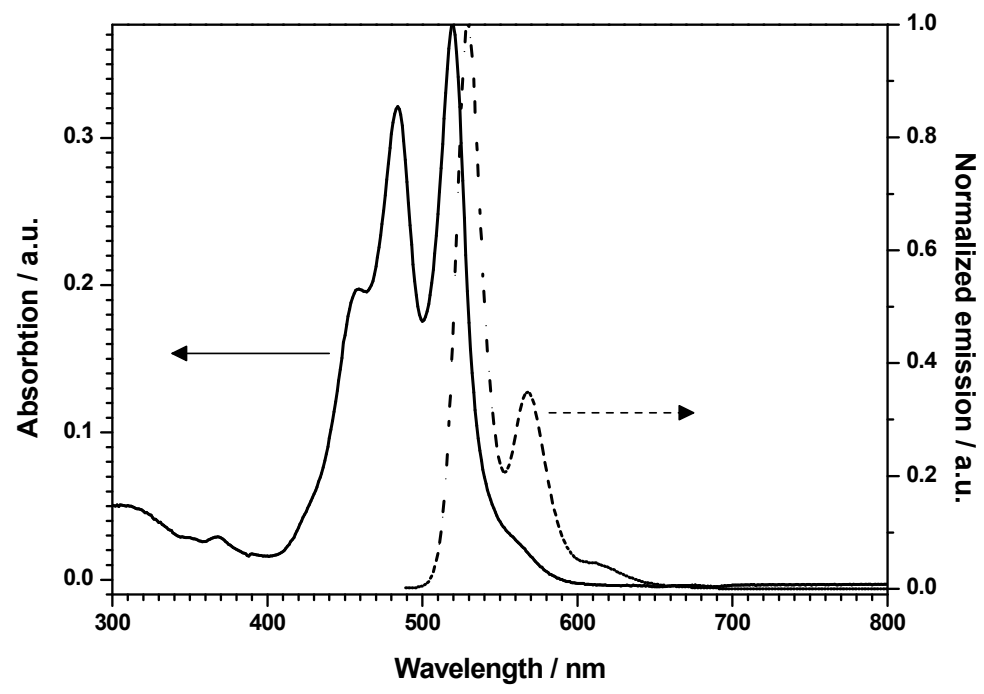

Figure 5: UV-vis absorption (solid line) and fluorescence (dotted line) spectra of PBI-PA in THF at room temperature. Concentration: $10^{-5} \mathrm{~mol} / \mathrm{L}$. 


\section{DEVICE FABRICATION AND MEASUREMENTS}

For the device fabrication bottom gate/bottom contact transistor substrates were used having an aluminum oxide layer as dielectric and patterned gold source and drain electrodes. After a UV-ozone treatment the substrates were immersed into a $10^{-5} \mathrm{~mol} / \mathrm{L}$ solution of PBI-PA in tetrahydrofuran (THF) under ambient conditions at room temperature. After 24 hours the substrates were rinsed with THF and baked on a hotplate at $110^{\circ} \mathrm{C}$ for 20 minutes to remove residual solvent. In Figure 6 an AFM height image captured inside a channel of a transistor is shown. A smooth surface comparable to the bare substrate is measured which is expected for a dense and homogenous monolayer. Some high islands of approximately $20 \mathrm{~nm}$ are also found which are supposed to be PBI-PA aggregates.
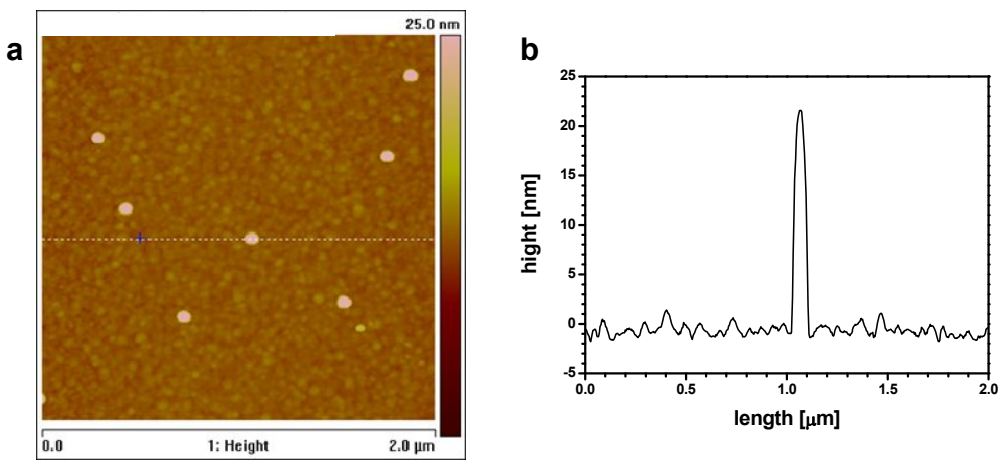

Figure 6: a) AFM height image of a PBI-PA SAMFET and b) the corresponding cross section.

To get information about the thickness X-ray photoelectron spectroscopy (XPS) and X-ray reflectivity (XRR) measurements were done. XPS studies show that a $3.1 \mathrm{~nm}$ thick organic layer is grown on top of the aluminum oxide dielectric. Additionally the XPS measurements revealed that the phosphorus is present within the whole organic layer. It is assumed that due to strong intermolecular $\pi$ - $\pi$-stacking of the perylene cores not all phosphonic acid groups react with the surface which suggests some kind of tilted configuration of the molecules. Further informations are given by XRR investigations which also pointed out that a smooth and $3.0 \mathrm{~nm}$ thick organic layer is assembled on top of the dielectric. For simulations of the XRR measurements, the layer is segmented into three different interlayer's having a thickness from top to the bottom of $0.71 \mathrm{~nm}, 0.98 \mathrm{~nm}$ and $1.32 \mathrm{~nm}$. XRR analysis also showed an enhanced electron density in the middle layer which suggests that here the aromatic core is present.

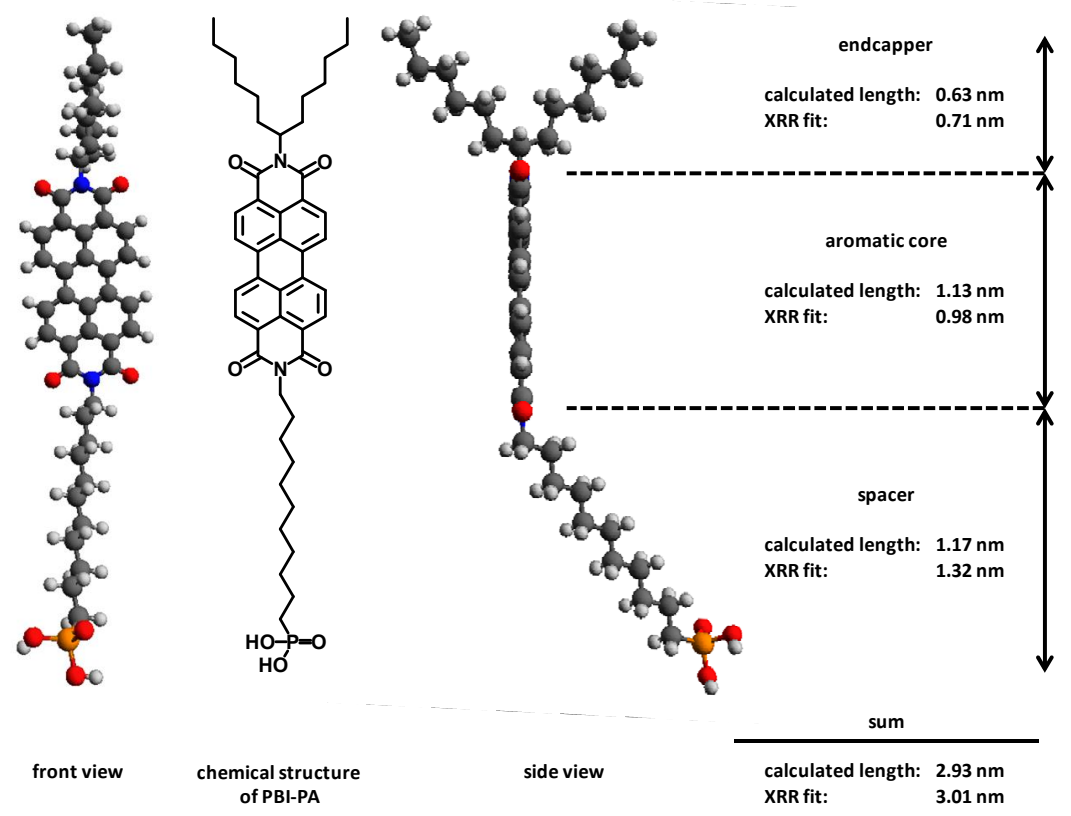

Figure 7: Calculated intramolecular distances of PBI-PA and results of XRR measurements. 
Molecular modeling using Avogadro 1.0.3 software with the united force-field (UFF) was done to get informations about the length of the different molecular units inside the PBI-PA molecule. As shown in figure 7 the result of the experimental XRR investigations matches quite well with the theoretical thickness of the monolayer. The in-plane order of the monolayer was investigated by grazing incidence X-ray (GIXD). A broad reflex at $\mathrm{q}_{\mathrm{xy}}=17.1 \mathrm{~nm}^{-1}$ was found which revealed a perylene core-core distance of $0.36 \mathrm{~nm}$. From the width of the diffraction peak $\left(\Delta \mathrm{q}_{\mathrm{xy}}\right)$ of $2 \mathrm{~nm}^{-1}$, the correlation length, or crystal size, was estimated to be $3.1 \mathrm{~nm}$. This length corresponds to 9 perylene cores.

SAMFETs have been prepared by immersing predefined transistor substrates with an aluminum oxide dielectric and gold electrodes into a solution of PBI-PA like described before. All measured transistors showed field-effect behavior. Mobilities up to $10^{-3} \mathrm{~cm}^{2} / \mathrm{Vs}$ and high on/off-ratios of $10^{5}$ were obtained. SAMFETs with channel length up to $100 \mu \mathrm{m}$ were obtained for the first time. The mobility increases with the channel length and saturates at a channel length of $40 \mu \mathrm{m}$ which is a fingerprint for SAMFETs with a completely covered surface ${ }^{[19]}$. Typical transfer and output characteristics are shown in Figure 8. It is remarkable that all of the measured transistors worked (device yield $=100 \%$ ). Also the fact that working SAMFETs can be prepared from a PBI-PA solution which has been stored for several weeks under ambient conditions demonstrates that PBI-PA is a robust molecule for the preparation of n-type SAMFETs.
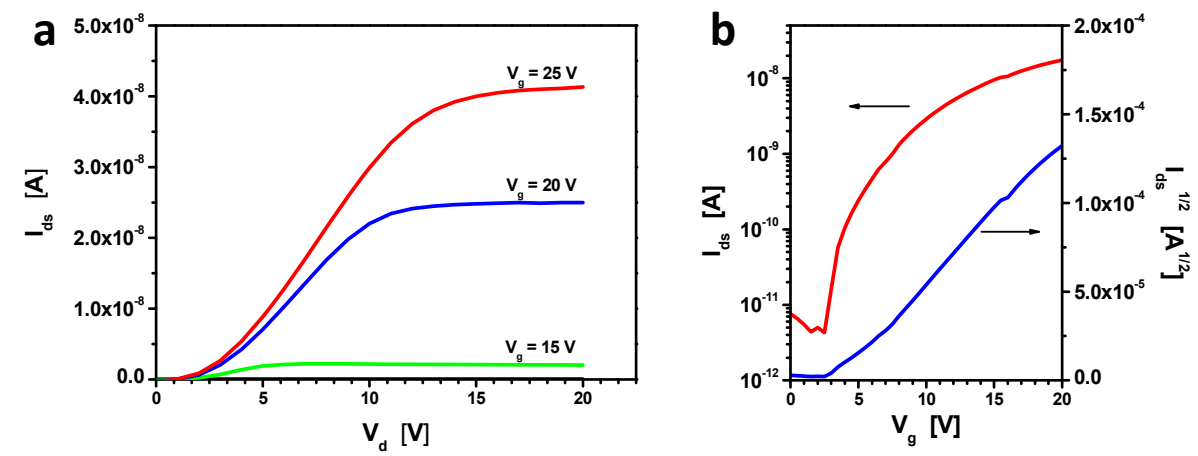

Figure 8: a) Transistor characteristics of a SAMFET based on PBI-PA with a channel length of $40 \mu \mathrm{m}$ and a channel width of $1000 \mu \mathrm{m}$. a) Output characteristics: the drain voltage is swept from $0 \mathrm{~V}$ to $20 \mathrm{~V}$, the gate voltage is varied starting from $0 \mathrm{~V}$ to $25 \mathrm{~V}$ in $5 \mathrm{~V}$ per step. b) Transfer characteristics of the device in the saturation regime with a drain voltage of $20 \mathrm{~V}$.

A bias inverter based on the complementary metal-oxide-semiconductor (CMOS) technology was build using a p-type and an n-type SAMFET on two substrates. The p-type SAMFET is based on a thiophene derivative connected covalently to silicon dioxide ${ }^{12}$. PBI-PA on aluminum oxide was used for the n-type SAMFET. Both SAMFETs were connected in a complementary inverter configuration as depicted in figure $9 \mathrm{~b}$. The resulting inverter characteristics are shown in figure $9 \mathrm{a}$. When the supply voltage $\left(\mathrm{V}_{\mathrm{dd}}\right)$ was set at $30 \mathrm{~V}$ and the input voltage $\left(\mathrm{V}_{\mathrm{in}}\right)$ was swept from $30 \mathrm{~V}$ to $0 \mathrm{~V}$, a high gain value of $\sim 15$ with the "trip-point" at around $23 \mathrm{~V}$ of $\mathrm{V}_{\text {in }}$ and a noise margin of $7 \mathrm{~V}$ was obtained.
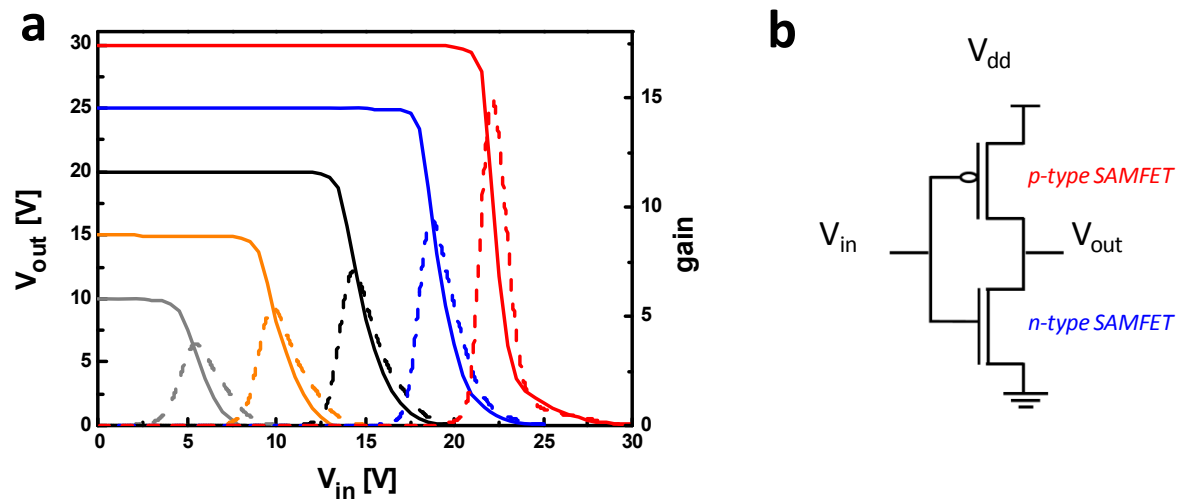

Figure 9: SAMFET based complementary inverter. (a) Device characteristics: The supply voltage $\left(\mathrm{V}_{\mathrm{dd}}\right)$ was varied starting from $10 \mathrm{~V}$ to $30 \mathrm{~V}$ in $5 \mathrm{~V}$ per step. The dashed lines present the gains. (b) Diagram of the bias inverter. 


\section{SUMMARY}

In summary we synthesized a new perylene bisimide, having a semiconducting perylene bisimide core, a branched alkyl tail on one side to increase solubility and on the other side a linear alkyl tail with a phosphonic acid anchor group. The phosphonic acid anchor group enables a covalent fixation of the perylene bisimide to aluminum oxide surfaces. With this substance we were able to fabricate highly reproducible monolayer field-effect transistors by simple self-assembly under ambient conditions. High on/off-ratios of $10^{5}$ and mobilities up to $10^{-3} \mathrm{~cm}^{2} / \mathrm{Vs}$ were achieved. Besides the transistors a CMOS bias inverter based solely on SAMFETs was realized for the first time and showed a large noise margin of 7 volts and a high gain of 15 .

\section{ACKNOWLEDGEMENT}

The work of Andreas Ringk and Fatemeh Gholamrezaie forms part of the research programme of the Dutch Polymer Institute (DPI), projects 624 and 627. We gratefully acknowledge Dr. C. van der Marel (Philips Research Laboratories, Eindhoven) for the XPS analysis and fruitful discussions. We are grateful to Bas van der Putten (Holst Centre) for technical assistance, to Ingo Salzmann (Humboldt University Berlin) for help with the X-ray measurements and the Austrian Science Foundation FWF:[P21094].

\section{REFERENCES}

[1] Whitesides, G. M. and Grzybowski, B., "Self-Assembly at All Scales," Science, 295, 2418-2421 (2002).

[2] Linford, M. R., Fenter, P., Eisenberger, P. M. and Chidsey, C. E. D, J., "Alkyl monolayers on silicon prepared from 1-alkenes and hydrogen-terminated silicon,” J. Am. Chem. Soc., 117, 3145-3155 (1995).

[3] O'Regan, B. and Grätzel, M., "A low-cost, high-efficiency solar cell based on dye-sensitized colloidal TiO 2 films," Nature, 353, 737-740 (1991).

[4] Besbes, S., Ltaief, A., Reybier, K., Ponsonnet, L., Jaffrezic, N., Davenas, J. and Ben Ouada, H., "Injection modifications by ITO functionalization with a self-assembled monolayer in OLEDs,” Syn. Met., 138, 197-200 (2003).

[5] Chua, L.-L., Zaumseil, J., Chang, J.-F. Ou, E. C.-W., Ho, P. K.-H., Sirringhaus, H. and Friend, R. H., “General observation of n-type field-effect behaviour in organic semiconductors," Nature, 434, 194-199 (2005).

[6] Novak, M., Schmaltz, T., Faber, H. and Marcus Halik, "Influence of self-assembled monolayer dielectrics on the morphology and performance of $\alpha, \omega$-dihexylquaterthiophene in thin film transistors" Appl. Phys. Lett., 98, 093302 (2011).

[7] Collet, J., Tharaud, O., Chapoton, A. and Vuillaume, D., "Low-voltage, 30 nm channel length, organic transistors with a self-assembled monolayer as gate insulating films," Appl. Phys. Lett., 76, 1941-1943 (2000).

[8] Cheng, X., Noh, Y.-Y., Wang, J., Tello, M., Frisch, J., Blum, R.-P., Vollmer, A., Rabe, J. P., Koch, N. and H. Sirringhaus, "Controlling Electron and Hole Charge Injection in Ambipolar Organic Field-Effect Transistors by Self-Assembled Monolayers" Adv. Funct. Mater, 19, 2407-2415 (2009).

[9] Dinelli, F., Murgia, M., Levy, P., Cavallini, M., Biscarini, F. and de Leeuw, D. M., "Spatially Correlated Charge Transport in Organic Thin Film Transistors," Phys. Rev. Lett., 92, 116802 (2004).

[10] Liu, S.-W., Lee, C.-C., Wen, J.-M. and Chen, C.-T., "In situ vacuum measurement of the thickness dependence of electron mobility in naphthalenetetracarboxylic diimide-based field-effect transistors," Appl. Phys. Lett., 98, 023306 (2011).

[11] Tulevski, G. S., Miao, Q., Fukuto, M., Abram, R., Ocko, B., Pindak,R., Steigerwald, M. L., Kagan, C. R. and Nuckolls, C. "Attaching organic semiconductors to gate oxides: In situ assembly of monolayer field effect transistors," J. Am. Chem. Soc., 126, 15048-15050 (2004).

[12] Smits, E. C. P., Mathijssen, S. G. J., van Hal, P. A., Setayesh, S., Geuns, T. C. T., Mutsaers, K. A. H. A., Cantatore, E., Wondergem, H. J., Werzer, O., Resel, R., Kemerink, M., Kirchmeyer, S., Muzafarov, A. M., 
Ponomarenko, S. A., de Boer, B., Blom, P. W. M. and de Leeuw, D. M., "Bottom-up organic integrated circuits, “Nature, 455, 956-959 (2008).

[13] de Leeuw , D. M., Simenon, M. M. J., Brown, A.R. and Einerhand, R. E. F., "Stability of n-type doped conducting polymers and consequences for polymeric microelectronic devices," Synthetic Metals, 87, 53-59 (1997).

[14] Novak, M., Ebel, A., Meyer-Friedrichsen, T., Jedaa, A., Vieweg, B. F., Yang, G., Voitchovsky, K., Stellacci, F., Spiecker, E., Hirsch, A. and Halik, M. "Low-voltage p- and n-type organic self-assembled monolayer field effect transistors," Nano Lett. 11, 156-159 (2011).

[15] Jones, B. A., Facchetti, A., Wasielewski, M. R. and Marks, T. J. "Tuning orbital energetics in arylene diimide semiconductors. Materials design for ambient stability of n-type charge transport," J. Am. Chem. Soc., 129, 15259-15278 (2007).

[16] Wicklein, A., Lang, A., Muth, M. and Thelakkat, M. "Swallow-tail substituted liquid crystalline perylene bisimides: Synthesis and thermotropic properties," J. Am. Chem. Soc., 131, 14442-14453 (2009).

[17] Ringk, A., Li, X., Gholamrezaie, F., Smits, E. C. P., Neuhold, A., Moser, A., Gelinck G. H., Resel, R., de Leeuw D. M. and Strohriegl, P., "N-type self-assembled monolayer field-effect transistors and complementary inverters" (submitted).

[18] Holman, M. W., Liu, R. and Adams, D. M., "Single-molecule spectroscopy of interfacial electron transfer," J. Am. Chem. Soc., 125, 12649-12654 (2003).

[19] Mathijssen, S. G. J., Smits, E. C. P., van Hal, P. A., Wondergem, H. J., Ponomarenko, S. A., Moser, A., Resel, R., Bobbert, P. A., Kemerink, M., Janssen, R. A. J. and Leeuw, D. M. de, "Monolayer coverage and channel length set the mobility in self-assembled monolayer field-effect transistors, "Nature Nanotech. 10, 674-680 (2009). 